\title{
Measurements and searches of Higgs boson decays to two fermions
}

\author{
Carlo Schiavi ${ }^{* \dagger}$ \\ Università degli Studi di Genova and INFN Genova \\ E-mail: carlo.schiavi@ge.infn.it
}

\begin{abstract}
In this report, we present the latest ATLAS measurements and searches of Higgs boson decays to two third- or two second-generation quarks or leptons, focusing on the $H \rightarrow \mu \mu, H \rightarrow b \bar{b}, H \rightarrow c \bar{c}$ channels. In addition, the search for $H \rightarrow \phi / \rho+\gamma$ decays is reported. All results are obtained using $36 \mathrm{fb}^{-1}$ of proton-proton collision data collected by the ATLAS experiment at the LHC, operating at a center-of-mass energy of $13 \mathrm{TeV}$.
\end{abstract}

XXVI International Workshop on Deep-Inelastic Scattering and Related Subjects (DIS2018) 16-20 April 2018

Kobe, Japan

* Speaker.

${ }^{\dagger}$ On behalf of the ATLAS Collaboration. 


\section{Introduction}

In 2012, analyzing proton-proton ( $p p$ ) collision data delivered by the Large Hadron Collider (LHC) [1] at $\sqrt{s}=7$ and $8 \mathrm{TeV}$, the ATLAS and CMS Collaborations observed a new particle with a mass of approximately $125 \mathrm{GeV}[2,3]$. The properties of the newly discovered particle were found to be compatible with those of a Standard Model (SM) Higgs boson and precise measurements of the coupling of the Higgs boson to other SM particles further strengthened that hypothesis. Those results are predominantly based on the study of the bosonic decay modes, $H \rightarrow \gamma \gamma, H \rightarrow Z Z^{*}$ and $H \rightarrow W W^{*}$.

To provide further insight into the nature of electroweak symmetry breaking, it is of particular importance to measure the Yukawa couplings between the Higgs boson and the fermions, testing the corresponding mass-coupling relation predicted by the SM. The ATLAS experiment [4] has searched the Higgs boson in different fermionic decay modes, namely $H \rightarrow \mu \mu, H \rightarrow \tau \tau, H \rightarrow b \bar{b}$ and $H \rightarrow c \bar{c}$. The searches are performed exploiting three production modes: gluon fusion $(g g \mathrm{~F})$, vector boson fusion $(\mathrm{VBF})$ and associated production with a vector boson $(V H)$. The $g g \mathrm{~F}$ mode has the largest cross section among all the production modes and, as an example, is used to study the $H \rightarrow \mu \mu$ decay mode, which has a very clean signature, but a low branching ratio. The VBF production mode has the second-largest cross section and is characterized by two forward VBF jets from light quarks, separated by a large rapidity gap from any other significant hadronic activity, due to absence of color connection between the Higgs boson and the VBF jets. The VBF mode is used to study basically all the fermionic decay modes investigated. Finally, the $V H$ production mode foresees a Higgs boson produced in association with a $W$ or $Z$ boson, providing a clean signature and an efficient trigger strategy by exploiting the leptonic decay of the vector bosons. The $V H$ mode is used to study the $H \rightarrow \tau \tau, H \rightarrow b \bar{b}$ and $H \rightarrow c \bar{c}$ decays.

In this report, we present the latest ATLAS results for the searches of the SM Higgs boson in the $H \rightarrow \mu \mu$ (Section 2), $H \rightarrow b \bar{b}$ (Section 3) and $H \rightarrow c \bar{c}$ (Section 4) decay modes, using $36 \mathrm{fb}^{-1}$ of $p p$ collision data collected at a center-of-mass energy of $13 \mathrm{TeV}$ in 2015 and 2016.

In addition, on the same data sample, the search for $H \rightarrow \phi / \rho+\gamma$ decays is reported (Section 5), aimed at probing Higgs boson couplings to the strange-quark, and the up- and down-quarks, respectively.

\section{2. $H \rightarrow \mu \mu$}

The $H \rightarrow \mu \mu$ decay has a clean final-state signature that allows to measure the Higgs coupling to second generation fermions. We report the results of a search [5] which targets both the $g g \mathrm{~F}$ and the VBF signatures. The inclusive signal region is formed by selecting dimuon events with exactly two opposite-charge muons. The dominant irreducible background is the $Z / \gamma^{*} \rightarrow \mu \mu$ (Drell-Yan) process. Figure 1 (left) shows the dimuon invariant mass $m_{\mu \mu}$ distributions for data and simulated events in the inclusive signal region. To enhance the analysis sensitivity, events in the inclusive signal region are classified into eight orthogonal categories. For events containing at least two jets, a Boosted Decision Tree (BDT) [6, 7] is trained to maximize the separation between the VBF signal and the total background. Two VBF categories, VBF tight and VBF loose, are defined from events with high BDT scores. The remaining events that are not selected for the VBF categories, 

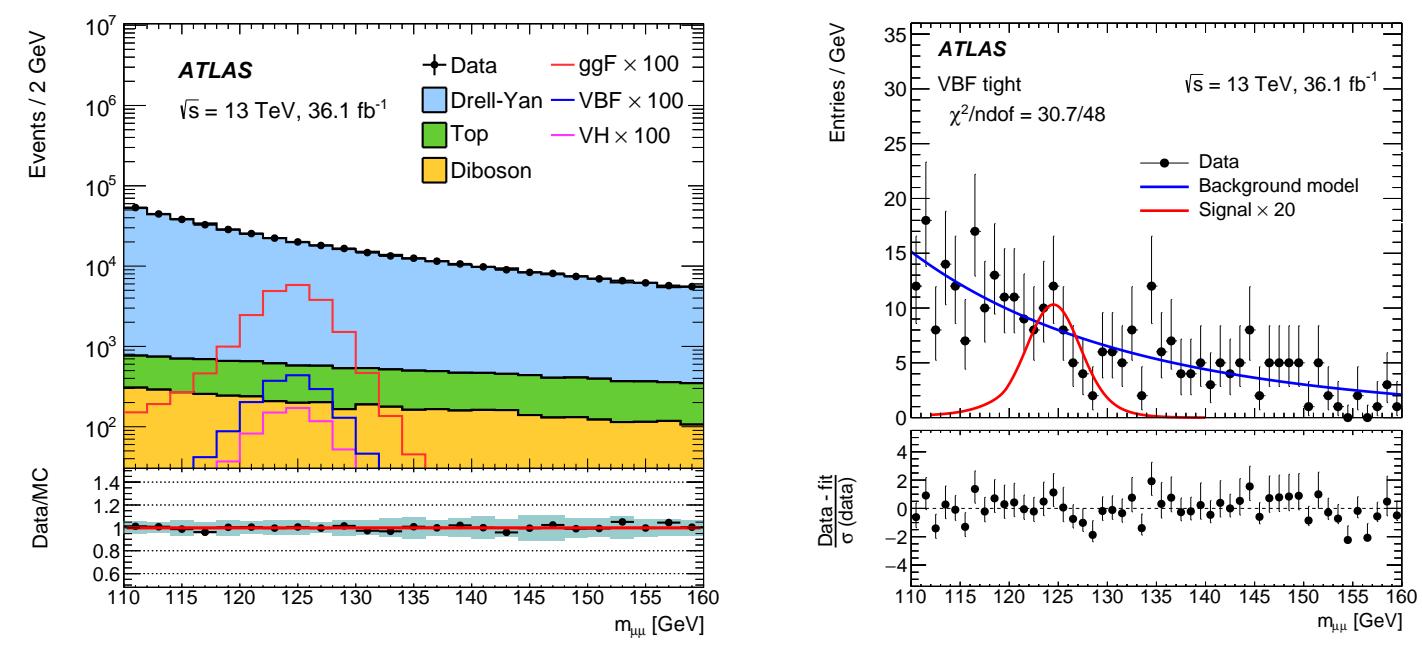

Figure 1: Observed and simulated $m_{\mu \mu}$ distributions in the inclusive signal region (left), and backgroundonly fit to the observed $m_{\mu \mu}$ distribution in the VBF tight category (right) [5].

including events with low BDT scores and events with less than two jets, all enter into the $g g \mathrm{~F}$ categories. These events are further divided into six categories according to the transverse momentum of the dimuon system (low, medium and high $p_{T}^{\mu \mu}$ categories) and the pseudorapidities of the muons (central and non-central categories). A binned maximum-likelihood fit to the observed $m_{\mu \mu}$ distribution in the range $110-160 \mathrm{GeV}$ is performed simultaneously in all categories to determine the background and extract the signal strength $\mu$, which is defined as the measured signal yield normalized to the SM prediction. No significant excess is observed in data. In Figure 1 (right), the observed $m_{\mu \mu}$ distribution in the most sensitive category, the VBF tight category, is compared to the background-only fit. The measured signal strength is $\mu=-0.1 \pm 1.5$. An upper limit on $\mu$ is computed using a modified frequentist CLs method $[8,9]$. The observed (expected) upper limit on $\mu$ at $95 \%$ confidence level (CL) is found to be 3.0 (3.1), driven by the data statistical uncertainty. When combined with the ATLAS Run 1 data, the measured signal strength is found to be $\mu=-0.1 \pm 1.4$, and the observed (expected) upper limit is 2.8 (2.9) at 95\% CL.

\section{3. $H \rightarrow b \bar{b}$}

The $H \rightarrow b \bar{b}$ decay is expected to have the largest branching ratio of $58 \%$ for a $125 \mathrm{GeV} \mathrm{SM}$ Higgs boson [10]. Measurements in this decay mode are crucial to constrain the overall Higgs boson decay width. However, an inclusive search in this decay mode is extremely challenging due to the overwhelming background from multi-jet production. We report the search ATLAS carried out targeting the associated production with a $W$ or $Z$ boson [11], where the leptonic decays of the vector boson lead to clean signatures for triggering, while rejecting most of the multi-jet background. The analysis is split into three channels, denoted by the number of charged leptons in the final states (0-, 1- and 2-lepton channels), corresponding to different decay modes of the vector boson: $Z H \rightarrow v v b \bar{b}, W H \rightarrow l v b \bar{b}$ and $Z H \rightarrow l l b \bar{b}$. Events are further split into regions based on the number of jets and the $p_{T}$ of the vector boson. The main background processes include contributions 
of multi-jet production from strong interactions, vector boson production with jets and top-quark productions. To maximize the sensitivity to the Higgs boson signal, a multivariate analysis (MVA) is setup making use of BDTs. In each signal region, a BDT is constructed, trained and evaluated separately with a set of observables encoding information on event kinematics and topology. The BDT discriminant from each signal region is used as the main observable in a global likelihood fit to extract the Higgs boson signal strength.

The observed signal strength for all channels combined is $\mu=1.20_{-0.23}^{+0.24}$ (stat.) ${ }_{-0.28}^{+0.34}$ (syst.), corresponding to an excess with a significance of $3.5 \sigma$, to be compared to the SM expectation of $3.0 \sigma$. Figure 2 (left) shows the data, background and signal yields where final-discriminant bins in all re-
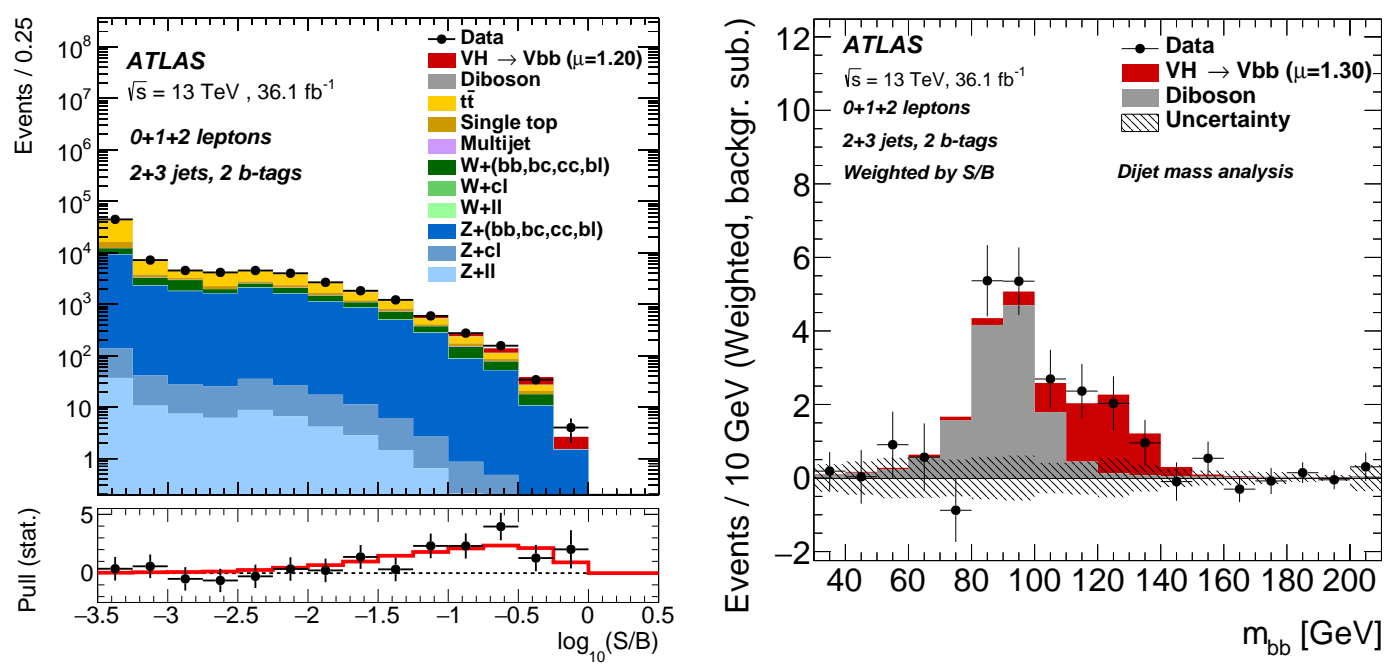

Figure 2: Data, signal and background yields from the MVA (left) and dijet-mass (right) $H \rightarrow b \bar{b}$ analysis [11].

gions are combined into bins of $\log (S / B)$ with $S$ and $B$ referring to the fitted signal and background yields, respectively. When combined with the ATLAS Run 1 data, the measured signal strength is $\mu=0.90 \pm 0.18$ (stat.) ${ }_{-0.19}^{+0.21}$ (syst.), corresponding to an excess with a significance of $3.6 \sigma$, to be compared to an expectation of $4.0 \sigma$.

The results of the MVA analysis are cross-checked by a diboson analysis and a dijet-mass analysis. In the diboson analysis, the $V Z$ signal strength instead of the $V H$ signal strength is extracted, using the same experimental method, to validate the MVA analysis results. The measured signal strength is $\mu_{V Z}=1.11_{-0.11}^{+0.12}$ (stat.) ${ }_{-0.19}^{+0.22}$ (syst.), in good agreement with the SM prediction. In the dijet-mass analysis, the BDT discriminant is replaced by the $m_{b \bar{b}}$ variable as the main observable in the global fit. The measured signal strength is $\mu=1.30_{-0.27}^{+0.28}$ (stat.) ${ }_{-0.29}^{+0.37}$ (syst.), in good agreement with the MVA result. The $m_{b \bar{b}}$ distribution for all regions summed, weighted by their respective $S / B$ after subtraction of all backgrounds except for the $W Z$ and $Z Z$ diboson processes, is shown in Figure 2 (right). The data and the sum of expected signal and background yields are in good agreement. 


\section{4. $H \rightarrow c \bar{c}$}

In the SM, the branching fraction for a Higgs boson with a mass of $125 \mathrm{GeV}$ to decay to a pair of charm quarks is predicted to be $2.9 \%$. Nevertheless, this channel is experimentally very challenging, given the difficult task of correctly separating charm jets from light and beauty jets. Another option was followed by the ATLAS collaboration in a previous search [12], indirectly looking for the decay of the Higgs boson to $c$-quarks via the decay to $J / \Psi$ and obtaining an upper limit on this smaller branching ratio of $1.5 \times 10^{-3}$ at $95 \%$ confidence level, which approximately corresponds to a limit of 220 times the expectation from the SM.

Here we report the first direct search performed by the ATLAS experiment for the decay of the Higgs boson to a pair of charm quarks [13], targeting the production in association with a $Z$ boson decaying to charged leptons, $Z H \rightarrow l l c \bar{c}$. The aim of this study is to characterize the sensitivity of the ATLAS experiment to Higgs boson decays to $c \bar{c}$ and to introduce this promising approach to study the couplings of the Higgs boson to second-generation quarks. Events are required to contain exactly two same-flavor leptons having opposite charge and with an invariant mass consistent with that of the $\mathrm{Z}$ boson, and at least two jets one or two of which are required to be identified as charm jets ( $c$-jets). Data are analyzed in four categories with different expected signal purities and the invariant mass of the dijet system, $m_{c \bar{c}}$, constructed using the two highest $p_{T}$ jets, is the discriminating variable in each category. These categories are defined based on the transverse momentum of the reconstructed $Z$ boson and the number of $c$-tags amongst the leading jets (either one or two).

At this point, a simultaneous binned maximum profile likelihood fit to $m_{c \bar{c}}$ in the four categories is used to extract the signal yield and estimate the normalization for one of the dominant background sources, $Z+$ jets. The observed (expected) upper limit is thus found to be $2.7 \mathrm{pb}\left(3.9_{-1.1}^{+2.1} \mathrm{pb}\right)$ at $95 \%$ $\mathrm{CL}$, corresponding to an observed (expected) upper limit on signal strength $\mu$ of $110\left(150_{-40}^{+80}\right)$ at 95\% CL.

\section{5. $H \rightarrow \phi / \rho+\gamma$}

Currently, the light $(u, d, s)$ quark couplings to the Higgs boson are loosely constrained by existing data on the total Higgs boson width, while the large multi-jet background at the LHC inhibits the study of such couplings with inclusive $H \rightarrow q \bar{q}$ decays. Rare exclusive decays of the Higgs boson into a light meson and a photon can be used to probe the couplings of the Higgs boson to light quarks and would allow a search for potential deviations from the SM prediction. Specifically, the observation of the Higgs boson decay to a $\phi$ or $\rho$ meson and a photon would provide sensitivity to its couplings to the strange-quark, and the up- and down-quarks, respectively.

We report a search for the decays of Higgs and $Z$ bosons into $\phi \gamma$ and $\rho \gamma$ [14], where the $\phi$ and $\rho$ mesons are reconstructed via their dominant decays into the $K^{+} K^{-}$and $\pi^{+} \pi^{-}$final states, respectively. The background model is derived using a fully data driven approach and validated in a number of control regions, including sidebands in the $K^{+} K^{-}$and $\pi^{+} \pi^{-}$mass distributions. No significant excess of events above the background expectations is found, while the observed $95 \%$ CL upper limits on the branching fractions for $H \rightarrow \phi+\gamma$ and $H \rightarrow \rho+\gamma$ decays are 208 and 52 times the expected SM branching fractions, respectively. 


\section{References}

[1] L. Evans, P. Bryant, Journal of Instrumentation 3, S08001 (2008)

[2] ATLAS Collaboration, Phys. Lett. B716, 1 (2012), 1207.7214

[3] CMS Collaboration, Phys. Lett. B716, 30 (2012), 1207.7235

[4] ATLAS Collaboration, Journal of Instrumentation 3, S08003 (2008)

[5] ATLAS Collaboration, Phys. Rev. Lett. 119, 051802 (2017), 1705.04582

[6] L. Breiman et al., Classification and Regression Trees (Wadsworth and Brooks, Monterey, CA, 1984)

[7] B.P. Roe et al., Nucl. Instrum. Meth. A543, 577 (2005), physics/0408124

[8] G. Cowan, K. Cranmer, E. Gross, O. Vitells, Eur. Phys. J. C71, 1554 (2011), [Erratum: Eur. Phys. J. C73 ,2501 (2013)], 1007.1727

[9] A.L. Read, Journal of Physics G: Nuclear and Particle Physics 28, 2693 (2002)

[10] A. Djouadi, J. Kalinowski, M. Spira, Comput. Phys. Commun. 108, 56 (1998), hep-ph/9704448

[11] ATLAS Collaboration, JHEP 12024 (2017), 1708.03299

[12] ATLAS Collaboration, Phys. Rev. Lett. 114, 121801 (2015)

[13] ATLAS Collaboration, Phys. Rev. Lett. 120211802 (2018)

[14] ATLAS Collaboration, (2017), 1712.02758 